\title{
Upregulation of SOX9 promotes cell proliferation, migration and invasion in lung adenocarcinoma
}

\author{
XIAOYING WANG ${ }^{1}$, YING JU ${ }^{2}$, MI ZHOU ${ }^{3}$, XIAOLI LIU $^{3}$ and CHENGJUN ZHOU ${ }^{1}$ \\ ${ }^{1}$ Department of Pathology, The Second Hospital of Shandong University, Jinan, Shandong 250033; \\ ${ }^{2}$ Department of Clinical Laboratory, Shandong Provincial Hospital Affiliated to Shandong University, Jinan, \\ Shandong 250021; ${ }^{3}$ Department of Clinical Laboratory, The Second Hospital of Shandong University, \\ Jinan, Shandong 250033, P.R. China
}

Received August 6, 2014; Accepted April 24, 2015

DOI: $10.3892 / \mathrm{ol} .2015 .3303$

\begin{abstract}
Sex determining region Y-box 9 (SOX9) is an important transcription factor in development and has been implicated in several types of cancer. Although the association between upregulation of SOX9 and lung adenocarcinoma (ADC) has been reported previously, the role of SOX9 in the proliferation, migration and invasion of cancer cells remains unclear. Therefore, in the present study, SOX9 expression was detected in 163 human lung adenocarcinoma tissues by immunohistochemistry and western blotting. It was found that the SOX9 protein was over-expressed in the majority of lung adenocarcinoma. The full-length human SOX9 plasmid was then transfected into the lung ADC A549 cell line. An MTT assay was used to investigate the role of SOX9 in cell proliferation. Scratch and extracellular matrix cell invasion assays were performed to investigate whether SOX9 promotes the migration and invasion of lung ADC cells. The results revealed that ectopic overexpression of SOX9 in the lung adenocarcinoma cell line resulted in a marked increase in cell proliferation, migration and invasion. Accordingly, knockdown of SOX9 by RNA interference resulted in the inhibition of cell growth, migration and invasion. The present data indicate that SOX9 may act as a novel marker for lung adenocarcinoma and perform an important role in cell proliferation, migration and invasion.
\end{abstract}

\section{Introduction}

Lung cancer is the main cause of all cancer-associated mortalities worldwide (1). Non-small cell lung cancer (NSCLC) accounts for almost $80 \%$ of all lung cancers, and $50 \%$ of NSCLC cases are classified as adenocarcinoma (ADC). There is a trend that the

Correspondence to: Dr Chengjun Zhou, Department of Pathology, The Second Hospital of Shandong University, 247 Beiyuan, Jinan, Shandong 250033, P.R. China

E-mail: wang_xy1896@163.com

Key words: lung adenocarcinoma, SOX9, migration, invasion, proliferation incidence of lung ADC is increasing worldwide, particularly in women $(2,3)$. In addition, ADC is the most common histological type of lung cancer that arises in never and former smokers $(4,5)$. Despite advances in treatment, the five-year survival rate for patients with all stages of NSCLC is only $15 \%$ (6). Due to the life-threatening nature of lung cancers, it is important to obtain an improved understanding of the underlying mechanisms with respect to the functional roles of the molecules involved in the development and advancement of the cancer (7). Although numerous markers have been reported using gene expression profiling analysis of lung cancers (8), the current understanding of lung cancer development and evolution remains unclear, and critical genes and molecular pathways involved in lung cancer development and progression require further exploration.

Sex determining region Y-box 9 (SOX9) is a transcription factor and member of the SOX family that is emerging as an important transcriptional regulator. SOX9 function was first identified as a key regulator in the development of cartilage and the testes, with mutations in SOX9 causing campomelic dysplasia and autosomal sex reversal $(9,10)$. Subsequently, it emerged that SOX9 is upregulated in several tumor types, including lung ADC, breast carcinoma, colorectal cancer and prostate cancer (11-14). Although an association between the upregulation of SOX9 and lung ADC has been reported (15), the role of SOX9 in the proliferation, migration and invasion of cancer cells remains unclear. In the present study, the expression of SOX9 was detected in 163 human ADC tissues, and it was found that SOX9 was upregulated in the majority of ADC tissues compared with the corresponding adjacent non-cancerous lung tissues. The present study also revealed that knockdown of SOX9 in lung ADC cell lines resulted in a marked decrease in the growth, migration and invasion of the cells. Accordingly, the ectopic expression of SOX9 may dramatically promote cell growth, migration and invasion. The present study offers evidence that SOX9 may act as a novel marker for lung ADC and play a role in cell proliferation, migration and invasion

\section{Materials and methods}

Patients and tissue specimens. In total, 163 paired ADC lung samples and adjacent non-tumorous lung tissues were obtained from patients undergoing surgical resection at The Second 
Hospital of Shandong University (Jinan, Shandong, China) between 2009 and 2013. Prior to the present study, patient consent and approval from the Institutional Research Ethics Committee at the Second Hospital of Shandong University (Jinan, China) were obtained to use these clinical materials for research purposes. Sections of the tissue samples were immediately frozen in liquid nitrogen until protein extraction. The remaining tissue samples were fixed in $4 \%$ formalin and embedded into wax blocks for immunohistochemistry.

Cell culture and DNA construction. The human lung ADC A549 cell line was obtained from the American Type Culture Collection. The cells were routinely cultured in Dulbecco's Modified Eagle Medium (DMEM; Invitrogen, Carlsbad, CA, USA) supplemented with $10 \%$ heat-inactivated fetal bovine serum (Invitrogen), $100 \mathrm{U} / \mathrm{ml}$ penicillin (Invitrogen) and $100 \mu \mathrm{g} / \mathrm{ml}$ streptomycin (Invitrogen) at $37^{\circ} \mathrm{C}$ in a humidified cell incubator with a $5 \% \mathrm{CO}_{2}$ atmosphere. The full-length human SOX9 plasmid was constructed as described by a previous study (11). The coding sequences of SOX9 in the EST clone were amplified by polymerase chain reaction, using the following primer sequences: Forward, 5'-GGATCCCAT GAATCTCCTGGACCCCT-3', and reverse, 5'-GAATTCTCA AGGTCGAGTGAGCTGTGTGT-3'. The sequences were then subcloned into a pCMV-Tag2V expression vector (Stratagene, Inc., La Jolla, CA, USA). Cell transfection was performed using Lipofectamine 2000 (Invitrogen).

Immunohistochemistry. Paraffin-embedded specimens were cut into $4-\mu \mathrm{m}$ slices and baked at $65^{\circ} \mathrm{C}$ for $30 \mathrm{~min}$. The sections were deparaffinized with xylenes and rehydrated. The sections were submerged into EDTA antigenic retrieval buffer and microwaved $(800 \mathrm{~W}, 15 \mathrm{~min})$ for antigenic retrieval. The sections were treated with $3 \%$ hydrogen peroxide in methanol to quench the endogenous peroxidase activity, followed by incubation in $1 \%$ bovine serum albumin to block non-specific binding. The slides were incubated with the mouse antihuman primary monoclonal antibody against SOX9 (1:500; Abcam, Cambridge, MA, USA) overnight at $4^{\circ} \mathrm{C}$, and incubated with a horseradish peroxidase (HRP)-conjugated goat anti-mouse secondary antibody (1:50, A0216, Shanghai Beyotime Biotechnology Co., Ltd., Shanghai, China) at room temperature for $1 \mathrm{~h}$. DAB was applied for color development. The immunoreactivity pattern and histological appearance of all tissues were examined and scored by two independent pathologists. Nuclear SOX9 staining was evaluated according to a previously described scoring system (16). Briefly, the staining intensity, which was scored as $0,1+, 2+$ or $3+$, and the proportion of positively stained tumor cells were recorded for each tissue. A final staining score was obtained from these two parameters, as follows: Negative, intensity of 0 ; weak staining, intensity of $1+$ in $\leq 70 \%$ of tumor cells or intensity of $2+$ in $\leq 30 \%$ of tumor cells; moderate staining, intensity of $1+$ in $>70 \%$ of tumor cells, intensity of $2+$ in $>30$ and $\leq 70 \%$ of tumor cells or intensity of $3+$ in $\leq 30 \%$ of tumor cells; and strong staining, intensity of $2+$ in $>70 \%$ of tumor cells or intensity of $3+$ in $>30 \%$ of tumor cells.

Western blotting. Cell and tissue lysates were prepared in RIPA buffer and cleared by centrifugation. In total, $40 \mu \mathrm{g}$ of protein lysate per lane was separated on 12\% SDS-PAGE gels and transferred onto nitrocellulose membranes (Millipore, Billerica, MA, USA). For immunodetection, the membranes were incubated with antibodies against SOX9 (Abcam). Signals from HRP-coupled secondary antibodies were generated by enhanced chemiluminescence solution (Amersham, Piscataway, NJ, USA) and were detected by exposure of the membranes to X-ray films (Kodak, Rochester, NY, USA). The relative signal intensity was quantified by densitometry using UVIphoto and the UVIsoft UVIband Application V97.04 software (Uvitec, Cambridge, UK).

Small interfering RNA. For the protocol using small interfering (si)RNA, pooled siRNA (SMARTpool; Dharmacon, Inc., Lafayette, CO, USA) was used to target SOX9 gene expression according to the manufacturer's instructions, using the following sequences: 5'-GGAACAACCCGUCUACACA-3'; 5'-GAACAAGCCGCACGUCAAG-3'; 5'-GACCUUCGAU GUCAACGAG-3'; and 5'-GGAAGUCGGUGAAGAACGG-3' (Dharmacon, Inc.).

Cell proliferation assay. Cells treated with various conditions were seeded onto a 96-well plate. Subsequent to overnight incubation, the medium was removed and $20 \mu 1 \mathrm{MTT}(5 \mathrm{mg} / \mathrm{ml}$; Sigma-Aldrich, St. Louis, MO, USA) was added to each well. The 96 -well plates were incubated at $37^{\circ} \mathrm{C}$ for $4 \mathrm{~h}$. The plates were centrifuged, and the formazan precipitates were dissolved in $150 \mu \mathrm{l}$ of dimethyl sulfoxide. The absorbance of the solution was measured at $490 \mathrm{~nm}$ using a MRX II absorbance reader (DYNEX Technologies, Chantilly, VA, USA).

Scratch assay. The lung ADC A549 cell line was transfected with the SOX9 plasmid or treated with SOX9 siRNA. The cells were plated in a 12-well plate and scratched with a p200 pipet tip to generate a cell-free zone $(20 \mathrm{~mm}$ in length and $700 \mu \mathrm{m}$ in width). The cells were then incubated in $1 \%$ serum culture medium to avoid further cell proliferation. The migration of cells into the cell-free zone was monitored after $24 \mathrm{~h}$ using a Leica DFC 420c camera (Leica Microsystems, Wetzlar, Germany) and the migration rates ( length of cell migration /migration time ) were determined. The relative migration activity was determined using the value of the negative control as 1 .

Invasion assay. The lung ADC A549 cell line was transfected with human SOX9 plasmids or treated with SOX9 siRNA. The invasion assays were then performed using the QCM ECMatrix Cell Invasion Assay kit (Millipore, Billerica, MA). Briefly, $\sim 2 \times 10^{4}$ of the cells were grown in the upper chamber in serum-free medium, with $20 \%$ serum medium in the bottom chamber. The invasive migration of cells through the extracellular matrix layer was then determined by crystal violet staining in the bottom of the upper chamber. The relative invasion activity was determined by the measurement of the optical density at $560 \mathrm{~nm}$ and using the value of the negative control as 1 .

Statistical analysis. Student's $t$-test was used to assess the significance of the differences between groups. All statistical analyses were performed using the SPSS statistical software, version 17.0 (SPSS, Chicago, IL, USA). P<0.05 was considered to indicate a statistically significant difference. 

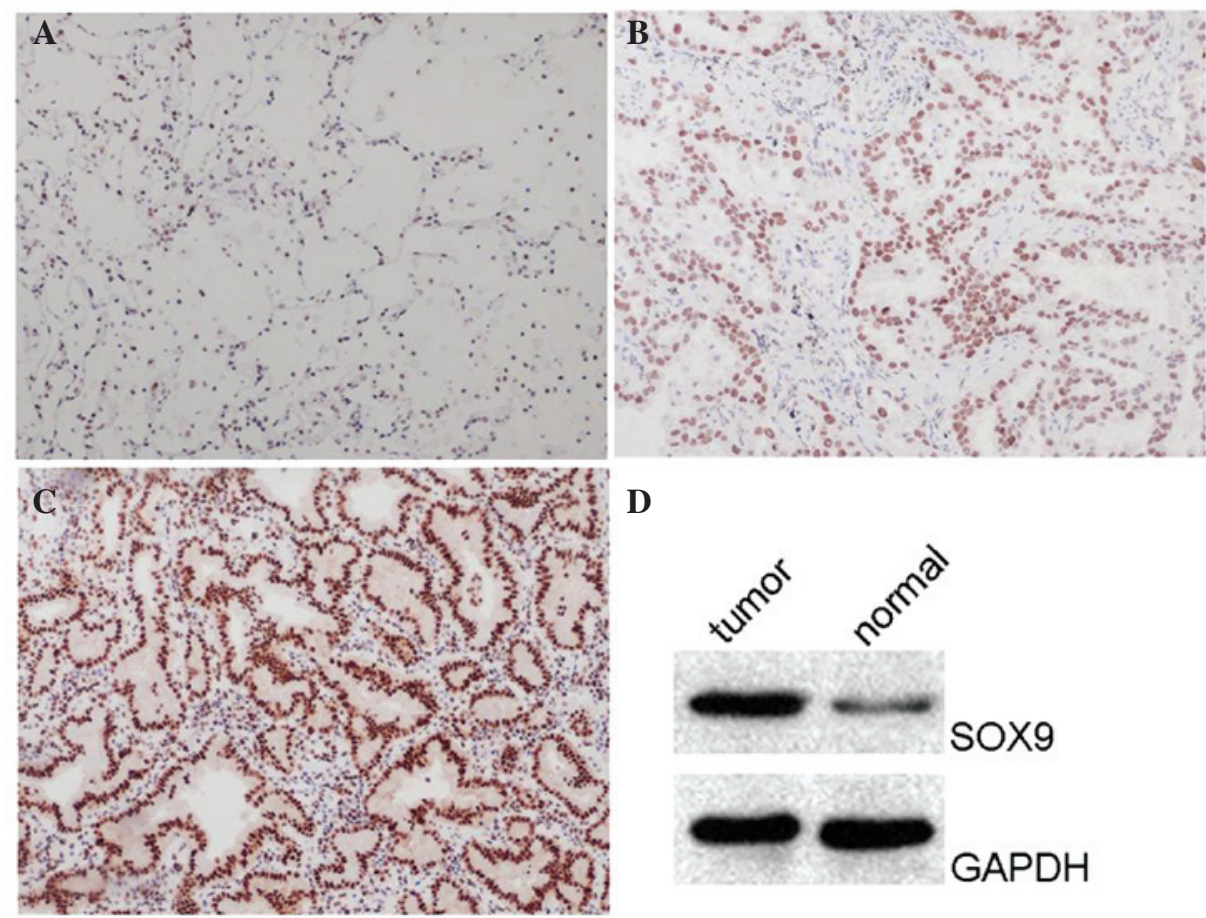

D

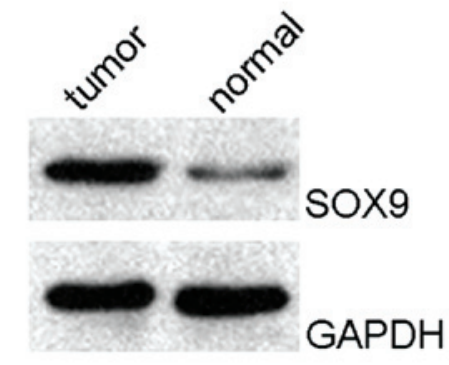

Figure 1. SOX9 protein expression in lung ADC samples and adjacent non-tumorous lung tissues. SOX9 expression was detected by immunohistochemistry. (A) Weak SOX9 expression was observed in all adjacent non-tumorous lung tissues. By contrast, the majority of lung ADC tissues demonstrated (B) moderate or (C) strong SOX9 expression $(\mathrm{P}<0.01)$. Magnifiction, $\mathrm{x} 100$. (D) The western blot analysis also revealed that the protein level of SOX9 was upregulated in cancerous tissues compared with the corresponding non-cancerous controls $(\mathrm{P}<0.01)$. SOX9, sex determining region Y-box 9; ADC, adenocarcinoma.

\section{Results}

Increased SOX9 protein expression in lung ADC tissues. To investigate the role of SOX9 in human lung ADC, the expression of SOX9 was compared between 163 lung ADC tissue samples and adjacent non-tumorous lung tissues by immunohistochemistry. Examples of SOX9 immunostaining are shown in Fig. 1. Weak SOX9 expression was observed in all adjacent non-tumorous lung tissues (Fig. 1A). By contrast, the majority of lung ADC tissues (59.7\%) demonstrated moderate (Fig. 1B) or strong (Fig. 1C) SOX9 expression $(\mathrm{P}<0.01)$. This result indicates that the SOX9 protein is over-expressed in the majority of lung ADC tissues. To further validate the hypothesis, western blot analysis was performed on the tissues, and revealed that the protein level of SOX9 was upregulated in cancerous tissues compared with the corresponding non-cancerous controls (Fig. 1D). This result was consistent with the data obtained from immunohistochemistry. The aforementioned results indicate that $\mathrm{SOX} 9$ protein expression is upregulated in the majority of lung ADC tissues.

Overexpression of SOX9 promotes cell proliferation and knockdown of SOX9 inhibits cell growth in the lung ADC cell line. Having established the association between SOX9 overexpression and lung ADC, the functional association between SOX9 expression and cancer phenotypes was assessed. The full-length human SOX9 plasmid or control was transfected into the lung ADC A549 cell line. After 2 days, the cells were reseeded and subjected to an MTT cell proliferation assay. Western blot analysis was also performed to confirm the overexpression of SOX9 (Fig. 2A). As revealed by the cell proliferation assay, the monolayer cell growth of A549 was significantly increased when SOX9 expression was upregulated (Fig. 2B). To further study the biological significance of SOX9 in cell growth, SOX9 expression was knocked down by RNA interference The A549 cell line was treated with SOX9 siRNA or non-targeting control siRNA and incubated for $48 \mathrm{~h}$ prior to performing the MTT assay. The silencing of SOX9 was confirmed by western blotting (Fig. 2C). The MTT assay revealed that knock-down of SOX9 resulted in a significant decrease of growth capability (Fig. 2D). The aforementioned results indicate that SOX9 is important for the proliferation of lung ADC cells.

Cell migration and invasion is promoted by overexpression of SOX9 and knockdown of SOX9 inhibits migration and invasion in the lung $A D C$ cell line. To investigate the effect of SOX9 on the migration and invasion of lung ADC cells, the A549 cells were transfected with the full-length human SOX9 plasmid or negative control (NC) and incubated for 2 days. To verify the over-expression of SOX9, western blotting was performed and the results revealed significantly increased expression of SOX9 in the A549 cells that were transfected with the SOX9 plasmid compared with the cells that were transfected wit the NC (Fig. 3A). The scratch assay was used to study the effect of SOX9 on A549 cell migration, and the result demonstrated that the ectopic expression of SOX9 may significantly promote cell migration compared with the control group (Fig. 3B). Similarly, the invasive capability of A549 cells transfected with the NC or SOX9 plasmid was evaluated by the ECMatrix cell invasion assay. As expected, the transfected SOX9 plasmid in A549 cells notably 

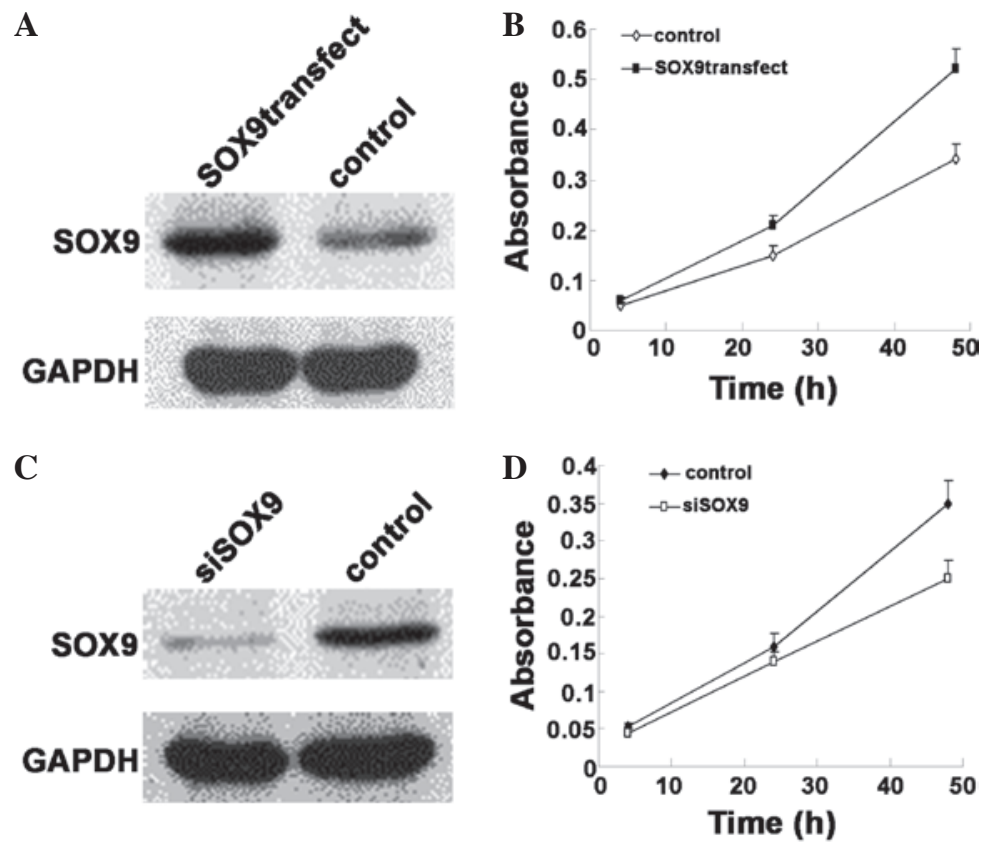

Figure 2. SOX9 is important for the proliferation of lung ADC cells. (A) The A549 cells were transfected with the full-length human SOX9 plasmid and the overexpression of SOX9 was confirmed by western blot analysis. (B) MTT assay revealed that the monolayer cell growth of A549 was significantly increased when SOX9 expression was upregulated. (C) SOX9 expression was also knocked down by RNA interference and the silencing of SOX9 was confirmed by western blotting. (D) An MTT assay revealed that knockdown of SOX9 resulted in a significant decrease of growth capability. ADC, adenocarcinoma; SOX9, sex determining region Y-box 9; siSOX9, small interfering RNA against SOX9; SOX9transfect, cells transfected with the SOX9 plasmid.
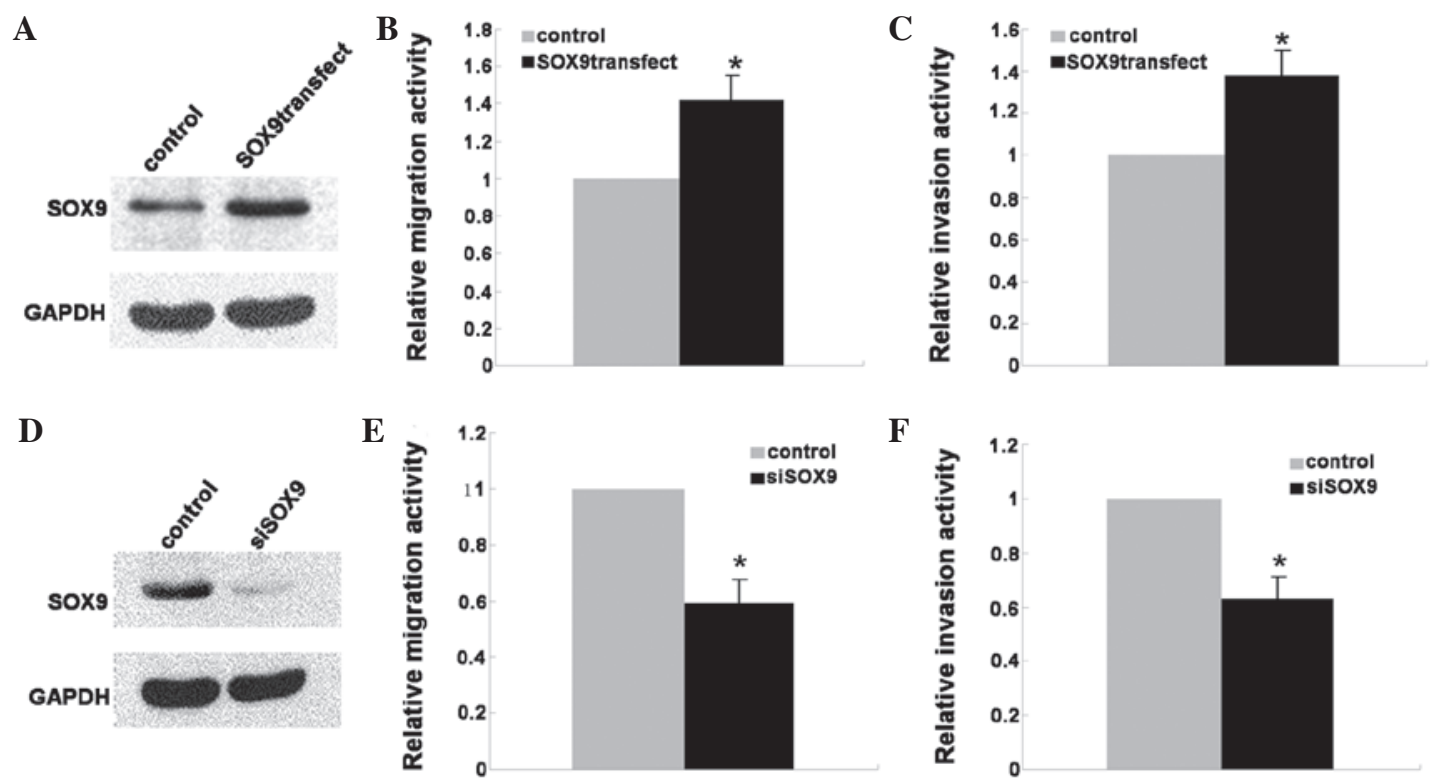

Figure 3. SOX9 is involved in the regulation of cell migration and invasion in the lung ADC cell line. (A) The A549 cells were transfected with the full-length human SOX9 plasmid or negative control plasmid and incubated for 2 days. The overexpression of SOX9 was confirmed by western blot analysis. (B) The migratory ability of A549 cells was promoted by overexpression of SOX9. The relative migration rates of A549 cells were determined by dividing the migration distance by the time. The migration rate of control cells was defined as 1. Statistical analysis was performed using Student's $t$-test. $\mathrm{P}<0.05$ was considered to indicate a statistically significant difference. (C) The invasive ability of the A549 cells was promoted by overexpression of SOX9. The results were expressed as relative values, using control cells as 1. (D) The A549 cells were treated with SOX9 siRNA or non-targeting control siRNA and incubated for 2 days. The silencing of SOX9 was confirmed by western blotting. (E) The migratory ability of A549 cells was suppressed as a result of SOX9 knockdown. The results were expressed as the relative values using cells treated with non-targeting control siRNA as 1. (F) The invasive ability of A549 cells was suppressed as a result of SOX9 knockdown. The results were expressed as relative values using cells treated with non-targeting control siRNA as 1 . "P<0.05. SOX9, sex determining region Y-box 9; siRNA, small interfering RNA; SOX9transfect, cells transfected with the SOX9 plasmid; siSOX9, siRNA against SOX9.

enhanced the invasive ability of the A549 cells compared with NC-transfected cells (Fig. 3C). Furthermore, the migratory and invasive capability of A549 cells transfected with siRNA of SOX9 or non-targeting control siRNA were evaluated by scratch and ECMatrix cell invasion assays. The results demonstrated that the silencing of SOX9 caused significant suppression of the migratory and invasive capability of A549 cells (Fig. 3D-F). 


\section{Discussion}

SOX9 was originally considered a chondrogenic transcription factor involved in bone formation and testis development, whereas the mutation of the SOX9 gene has been associated with campomelic dysplasia and autosomal sex reversal (17). Subsequent studies revealed SOX9 to be a multifaceted transcription factor that is vital for the development of numerous other organs and tissues, including the pancreas $(18,19)$, the prostate $(20,21)$, the intestine (22) and pigment cells (23). It has been well reported that genes and pathways critical for development may also perform important roles in cancer development and progression, and it is therefore not notable that SOX9 is associated with cancer. At present, SOX9 has been found to be upregulated in several tumor types, including lung ADC, breast carcinoma, colorectal cancer and prostate cancer (11-14). Despite the previously reported association between upregulation of SOX9 and lung ADC, the role of SOX9 in cancer cell proliferation, migration and invasion remains unclear (11).

The present study described the SOX9 expression in 163 lung ADC tissue samples and adjacent non-tumorous lung tissues. The data from immunohistochemistry revealed that weak SOX9 expression was observed in all adjacent non-tumorous lung tissues. By contrast, the majority of lung ADC (59.7\%) tissues demonstrated moderate or strong SOX9 expression $(\mathrm{P}<0.01)$. The western blotting analysis also revealed that SOX9 was upregulated in cancerous tissues compared with the corresponding non-cancerous controls. The differential expression of SOX9 between the lung ADC tissues and controls suggested that SOX9 may have some functional roles in cancer phenotypes. To assess this hypothesis, MTT, scratch and invasion assays were performed in the lung ADC A549 cell line. The results revealed that the overexpression of SOX9 promotes cell proliferation, migration and invasion. Accordingly, knockdown of SOX9 resulted in the inhibition of cell growth, migration and invasion. Based on the findings from the present study, it was hypothesized that SOX9, which is involved in the regulation of cell proliferation, migration and invasion, may act as a novel marker for lung ADC. Despite the present and previous studies, the precise pathway that SOX9 uses to promote proliferation, migration and invasion in lung ADC cells remains unclear. Additional investigation is required to delineate the pathway and mechanism underlying this involvement, and this may aid the understanding of lung cancer formation and identification of potential novel targets for cancer therapy.

\section{Acknowledgements}

This study was supported by grants from the National Natural Science Foundation of China (81000731) and the Promotive Research Fund for Excellent Young and Middle-Aged Scientists of Shandong Province (BS2010YY045).

\section{References}

1. Greenlee RT, Hill-Harmon MB, Murray T and Thun M: Cancer statistics, 2001. CA Cancer J Clin 51: 15-36, 2001.
2. Chen KY, Chang CH, Yu CJ, Kuo SH and Yang PC: Distribution according to histologic type and outcome by gender and age group in Taiwanese patients with lung carcinoma. Cancer 103: 2566-2574, 2005.

3. Yoshimi I, Ohshima A, Ajiki W, Tsukuma H and Sobue T: A comparison of trends in the incidence rate of lung cancer by histological type in the Osaka cancer registry, Japan and in the Surveillance, Epidemiology and End Results Program, USA. Jpn J Clin Oncol 33: 98-104, 2003.

4. Toh CK and Lim WT: Lung cancer in never-smokers. J Clin Pathol 60: 337-340, 2007.

5. Liu NS, Spitz MR, Kemp BL, Cooksley C, Fossella FV, et al: Adenocarcinoma of the lung in young patients: The M. D. Anderson experience. Cancer 88: 1837-1841, 2000.

6. Chan BA and Hughes BG: Targeted therapy for non-small cell lung cancer: Current standards and the promise of the future. Transl Lung Cancer Res 4: 36-54, 2015.

7. Jemal A, Siegel R, Xu J and Ward E: Cancer statistics, 2010. CA Cancer J Clin 60: 277-300, 2010.

8. Roh MS: Molecular pathology of lung cancer: Current status and future directions. Tuberc Respir Dis (Seoul) 77: 49-54, 2014.

9. Wagner T, Wirth J, Meyer J, Zabel B, Held M, et al: Autosomal sex reversal and campomelic dysplasia are caused by mutations in and around the SRY-related gene SOX9. Cell 79: 1111-1120, 1994.

10. Foster JW, Dominguez-Steglich MA, Guioli S, Kwok C, Weller PA, et al: Campomelic dysplasia and autosomal sex reversal caused by mutations in an SRY-related gene. Nature 372: 525-530, 1994.

11. Jiang SS, Fang WT, Hou YH, Huang SF, Yen BL, et al: Upregulation of SOX9 in lung adenocarcinoma and its involvement in the regulation of cell growth and tumorigenicity. Clin Cancer Res 16: 4363-4373, 2010.

12. Müller P, Crofts JD, Newman BS, Bridgewater LC, Lin CY, et al: SOX9 mediates the retinoic acid-induced HES-1 gene expression in human breast cancer cells. Breast Cancer Res Treat 120: 317-326, 2010.

13. Lü B,Fang Y,Xu J,Wang L, Xu F, etal: Analysis of SOX9 expression in colorectal cancer. Am J Clin Pathol 130: 897-904, 2008.

14. Wang H, Leav I, Ibaragi S, Wegner M, Hu GF, et al: SOX9 is expressed in human fetal prostate epithelium and enhances prostate cancer invasion. Cancer Res 68: 1625-1630, 2008.

15. Zhou CH, Ye LP, Ye SX, et al: Clinical significance of SOX9 in human non-small cell lung cancer progression and overall patient survival. J Exp Clin Cancer Res 31: 18, 2012.

16. Sirma H, Broemel M, Stumm L, Tsourlakis T, Steurer S, et al: Loss of CDKN1B/p27Kip1 expression is associated with ERG fusion-negative prostate cancer, but is unrelated to patient prognosis. Oncol Lett 6: 1245-1252, 2013.

17. Südbeck P, Schmitz ML, Baeuerle PA and Scherer G: Sex reversal by loss of the $\mathrm{C}$-terminal transactivation domain of human SOX9. Nat Genet 13: 230-232, 1996.

18. Lynn FC, Smith SB, Wilson ME, Yang KY, Nekrep N and German MS: Sox 9 coordinates a transcriptional network in pancreatic progenitor cells. Proc Natl Acad Sci USA 104: 10500-10505, 2007.

19. Piper K, Ball SG, Keeling JW, Mansoor S, Wilson DI and Hanley NA: Novel SOX9 expression during human pancreas development correlates to abnormalities in Campomelic dysplasia. Mech Dev 116: 223-226, 2002.

20. Thomsen MK, Butler CM, Shen MM and Swain A: Sox9 is required for prostate development. Dev Biol 316: 302-311, 2008.

21. Wang H, McKnight NC, Zhang T, Lu ML, Balk SP and Yuan X: SOX9 is expressed in normal prostate basal cells and regulates androgen receptor expression in prostate cancer cells. Cancer Res 67: 528-536, 2007.

22. Blache P, van de Wetering M, Duluc I, Domon C, Berta P, et al: SOX9 is an intestine crypt transcription factor, is regulated by the Wnt pathway, and represses the CDX2 and MUC2 genes. J Cell Biol 166: 37-47, 2004.

23. Passeron T, Valencia JC, Bertolotto C, Hoashi T, Le Pape E, et al: SOX9 is a key player in ultraviolet B-induced melanocyte differentiation and pigmentation. Proc Natl Acad Sci USA 104: 13984-13989, 2007. 